Fixed Point Theory, 22(2021), No. 1, 157-168

DOI: $10.24193 /$ fpt-ro.2021.1.11

http://www.math.ubbcluj.ro/ nodeacj/sfptcj.html

\title{
FIXED AND PERIODIC POINT RESULTS IN CONE $b$-METRIC SPACES OVER BANACH ALGEBRAS; A SURVEY
}

\author{
ATAOLLAH ARABNIA FIROZJAH, HAMIDREZA RAHIMI, GHASEM SOLEIMANI RAD
}

\author{
Department of Mathematics, Faculty of Science, Central Tehran Branch, \\ Islamic Azad University, P.O. Box 13185/768, Tehran, Iran \\ E-mail: ata.arabniafirozjah.sci@iauctb.ac.ir; \\ rahimi@iauctb.ac.ir (Corresponding author); \\ gha.soleimani.sci@iauctb.ac.ir
}

\begin{abstract}
In this paper, we consider the concept of cone $b$-metric spaces over Banach algebras and obtain some fixed point results for various definitions of contractive mappings. Moreover, we discuss about the property $\mathrm{P}$ and the property $\mathrm{Q}$ of fixed point problems. Our results are significant, since we omit the assumptions of normality of cones under which can be generalized and unified a number of recently announced results in the existing literature. In particular, we refer to the results of Huang et al. [H. Huang, G. Deng, S. Radenović, Some topological properties and fixed point results in cone metric spaces over Banach algebras, Positivity. (2018), in press].

Key Words and Phrases: Cone $b$-metric space, Banach algebra, spectral radius, solid cone, fixed point.
\end{abstract}

2020 Mathematics Subject Classification: 47H10, 54H25, 47H09, 46J45.

\section{REFERENCES}

[1] M. Abbas, G. Jungck, Common fixed point results for noncommuting mappings without continuity in cone metric spaces, J. Math. Anal. Appl., 341(2008), 416-420.

[2] M. Abbas, B.E. Rhoades, Fixed and periodic point results in cone metric spaces, Appl. Math. Lett., 22(2009), 511-515.

[3] A. Ahmed, J.N. Salunke, Algebra cone generalized b-metric space over Banach algebra and fixed point theorems of generalized Lipschitz mappings, Asian-European J. Math., 11(2018), no. 3, 1850068:11 pages.

[4] I. Altun, B. Damjanović, D. Djorić, Fixed point and common fixed point theorems on ordered cone metric spaces, Appl. Math. Lett., 23(2010), 310-316.

[5] I.A. Bakhtin, The contraction mapping principle in almost metric space, Funct. Anal., 30(1989), 26-37.

[6] S. Banach, Sur les opérations dans les ensembles abstraits et leur application aux equations intégrales, Fund. Math. J., 3(1922), 133-181.

[7] A.S. Ćvetković, M.P. Stanić, S. Dimitrijević, S. Simić, Common fixed point theorems for four mappings on cone metric type space, Fixed Point Theory Appl., 589725(2011), 15 pages.

[8] S. Czerwik, Contraction mappings in b-metric spaces, Acta Math. Inform. Univ. Ostrav., 1(1993), no. 1, 5-11.

[9] K. Deimling, Nonlinear Functional Analysis, Springer-Verlag, 1985. 
[10] W.S. Du, A note on cone metric fixed point theory and its equivalence, Nonlinear Anal., 72(2010), no. 5, 2259-2261.

[11] W.S. Du, E. Karapinar, A note on b-cone metric and its related results: generalizations or equivalence?, Fixed Point Theory Appl., 210(2013), 7 pages.

[12] H. Huang, G. Deng, S. Radenović, Some topological properties and fixed point results in cone metric spaces over Banach algebras, Positivity, (2018), https://doi.org/10.1007/s11117-0180590-5.

[13] H. Huang, S. Hu, B.Z. Popovic, S. Radenović, Common fixed point theorems for four mappings on cone b-metric spaces over Banach algebras, J. Nonlinear Sci. Appl., 9(2016), 3655-3671.

[14] H.P. Huang, S. Radenović, Some fixed point results of generalized Lipschitz mappings on cone b-metric spaces over Banach algebras, J. Comput. Anal. Appl., 20(2016), 566-583.

[15] H. Huang, S. Radenović, G. Deng, A sharp generalization on cone b-metric space over Banach algebra, J. Nonlinear Sci. Appl., 10(2017), 429-435.

[16] H.P. Huang, S.Y. Xu, Correction: Fixed point theorems of contractive mappings in cone bmetric spaces and applications, Fixed Point Theory Appl., 55(2014), 5 pages.

[17] H. Huang, S. Xu, H. Liu, S. Radenović, Fixed point theorems and T-stability of Picard iteration for generalized Lipschitz mappings in cone metric spaces over Banach algebras, J. Comput. Anal. Appl., 20(2016), 869-888.

[18] L.G. Huang, X. Zhang, Cone metric spaces and fixed point theorems of contractive mappings, J. Math. Anal. Appl., 332(2007), 1467-1475.

[19] N. Hussain, M.H. Shah, KKM mappings in cone b-metric spaces, Comput. Math. Appl., 62(2011), 1677-1684.

[20] S. Janković, Z. Kadelburg, S. Radenović, On cone metric spaces, a survey, Nonlinear Anal., 74(2011), 2591-2601.

[21] G.S. Jeong, B.E. Rhoades, Maps for which $F(T)=F\left(T^{n}\right)$, Fixed Point Theory, 6(2005), 87-131.

[22] Z. Kadelburg, Lj. Paunović, S. Radenović, G. Soleimani Rad, Non-normal cone metric and cone b-metric spaces and fixed point results, Ser. A: Appl. Math. Inform. Mech., 8(2016), no. 2, $177-186$.

[23] M.A. Khamsi, N. Hussain, KKM mappings in metric type spaces, Nonlinear Anal., 73(2010), 3123-3129.

[24] P. Kumam, H. Rahimi, G. Soleimani Rad, The existence of fixed and periodic point theorems in cone metric type spaces, J. Nonlinear Sci. Appl., 7(2014), 255-263.

[25] H. Liu, S. Xu, Cone metric spaces with Banach algebras and fixed point theorems of generalized Lipschitz mappings, Fixed Point Theory Appl., 320(2013), 10 pages.

[26] H. Rahimi, B.E. Rhoades, S. Radenović, G. Soleimani Rad, Fixed and periodic point theorems for T-contractions on cone metric spaces, Filomat, 27(2013), no. 5, 881-888.

[27] H. Rahimi, P. Vetro, G. Soleimani Rad, Some common fixed point results for weakly compatible mappings in cone metric type space, Miskolc. Math. Notes, 14(2013), no. 1, 233-243.

[28] S. Reich, Some remarks concerning contraction mappings, Canad. Math. Bull., 14(1971), 121124.

[29] B.E. Rhoades, A comparison of various definition of contractive mappings, Trans. Amer. Math. Soc., 266(1977), 257-290.

[30] W. Rudin, Functional Analysis, McGraw-Hill, New York, 1991.

[31] I.A. Rus, Results and problems in the metrical common fixed point theory, Mathematica, 21(1979), no. 2, 189-194.

[32] I.A. Rus, Some variants of contraction principle, generalizations and applications, Stud. Univ. Babeş-Bolyai Math., 61(2016), no. 3, 343-358.

[33] Sh. Xu, S. Radenović, Fixed point theorems of generalized Lipschitz mappings on cone metric spaces over Banach algebras without assumption of normality, Fixed Point Theory Appl., 102(2014), 12 pages.

[34] Q. Yan, J. Yin, T. Wang, Fixed point and common fixed point theorems on ordered cone metric spaces over Banach algebras, J. Nonlinear Sci. Appl., 9(2016), 1581-1589.

[35] P.P. Zabrejko, K-metric and K-normed linear spaces: survey, Collect. Math., 48(1997), 825859. 
Received: February 14, 2019; Accepted: May 7, 2019. 